\title{
IgG4-related Disease Involving the Cardiovascular System: An Intracardiac Mass and a Mass Lesion Surrounding a Coronary Artery
}

\author{
Ryotaro Maeda ${ }^{1}$, Daisuke Naito ${ }^{2}$, Atsuo Adachi ${ }^{2}$, Hirokazu Shiraishi ${ }^{1}$, \\ Takashi Sakamoto $^{2}$ and Satoaki Matoba ${ }^{1}$
}

\begin{abstract}
:
A 61-year-old Japanese man with IgG4-related autoimmune pancreatitis developed a mass in the right atrium (RA) and a mass lesion surrounding the left anterior descending coronary artery. We performed an intracardiac echo catheter-guided percutaneous biopsy of the RA mass, and histologically diagnosed it as IgG4related disease. Oral corticosteroid therapy gradually downsized the mass lesions. We encountered a very rare case with mass lesions in the cardiovascular system of the IgG4-related disease that were able to be diagnosed using an intracardiac echo-guided biopsy.
\end{abstract}

Key words: IgG4-related disease, intracardiac mass, intracardiac echo, computed tomography angiography, fluorodeoxyglucose positron emission tomography

(Intern Med 58: 2363-2366, 2019)

(DOI: 10.2169/internalmedicine.2509-18)

\section{Introduction}

Immunoglobulin G4 (IgG4)-related disease is a systemic inflammatory disorder that is characterized by the elevation of the serum IgG4 concentration and the infiltration of IgG4-positive plasma cells into target organs. However, its involvement in the cardiovascular system is exceedingly rare and difficult to diagnose. Therefore, there is no consensus concerning the optimal therapy for the cardiovascular lesions. We herein report the case of a 61-year-old Japanese man with IgG4-related autoimmune pancreatitis who developed a mass in the right atrium (RA) and a mass lesion surrounding the left anterior descending coronary artery. We performed an intracardiac echo catheter-guided percutaneous biopsy of the RA mass and histologically diagnosed it as IgG4-related disease. After the administration of oral corticosteroid, the serum IgG4 levels decreased, and the RA and peri-coronary mass lesion gradually shrink in size. To our knowledge, this is the first report in which lesions in the cardiovascular system of the IgG4-related disease were his- tologically diagnosed using an intracardiac echo-guided biopsy.

\section{Case Report}

A 61-year-old Japanese man was admitted to our institution due to jaundice and fatigue. He had no history of autoimmune disease or any other underlying diseases. He had experienced neither chest pain nor dyspnea to suggest angina pectoris or pulmonary embolism. The findings of laboratory examinations on admission were as follows: total bilirubin: $12.0 \mathrm{mg} / \mathrm{dL}$, direct bilirubin: $9.2 \mathrm{mg} / \mathrm{dL}$, alkaline phosphatase: 1,417 IU/L, and $\gamma$-glutamyl transformer peptidase: $415 \mathrm{IU} / \mathrm{L}$. Immunology-related data were as follows: IgG: $2,835 \mathrm{mg} / \mathrm{dL}, \operatorname{IgG} 4: 1,440 \mathrm{mg} / \mathrm{dL}, \mathrm{IgE}: 514 \mathrm{IU} / \mathrm{mL}$, IgA: $114 \mathrm{mg} / \mathrm{dL}$, IgM: $61 \mathrm{mg} / \mathrm{dL}$, soluble interleukin-2 receptor: $488 \mathrm{U} / \mathrm{mL}$, and antinuclear antibodies: negative. Abdominal computed tomography showed a diffusely enlarged "sausage-like" swollen pancreas occluding the common bile duct, leading to occlusive jaundice (Fig. 1A). Endoscopic ultrasound-guided fine-needle aspiration histologically

${ }^{1}$ Department of Cardiovascular Medicine, Graduate School of Medical Science, Kyoto Prefectural University of Medicine, Japan and ${ }^{2}$ Department of Cardiology, Fukuchiyama City Hospital, Japan

Received: December 14, 2018; Accepted: February 13, 2019; Advance Publication by J-STAGE: May 22, 2019

Correspondence to Dr. Ryotaro Maeda, ryo-m@koto.kpu-m.ac.jp 

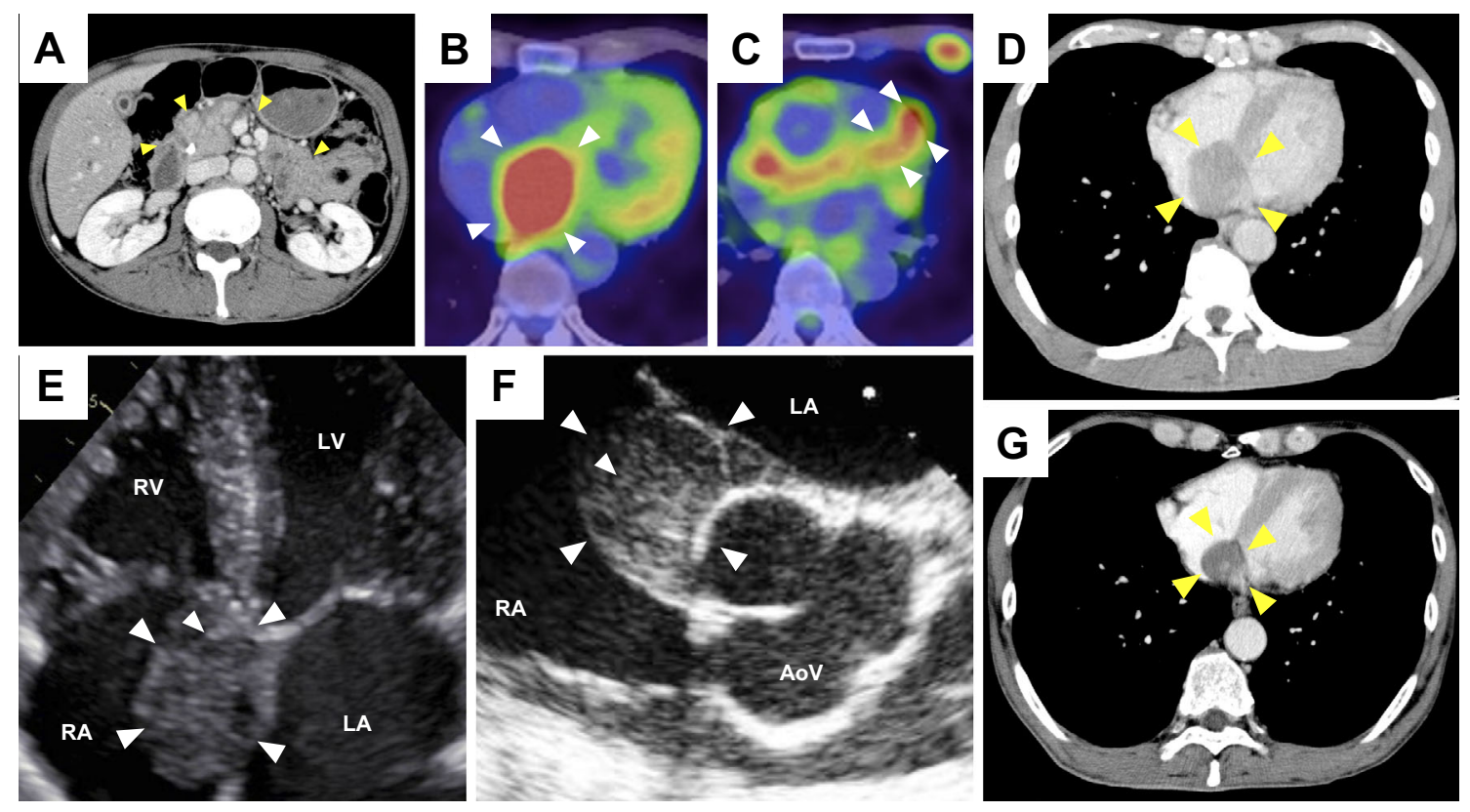

Figure 1. (A) Contrast-enhanced abdominal computed tomography shows a "sausage-like" swollen pancreas (arrowheads). (B and C) Fluorodeoxyglucose positron emission tomography shows an increased uptake in the RA mass and interventricular septum (arrowheads). (D) Contrast-enhanced thoracic computed tomography shows a mass occupying the RA (arrowheads). (E and F) Transthoracic and transesophageal echocardiography show a mass extending from the interatrial septum into the RA (arrowheads). (G) Follow-up thoracic computed tomography shows that the RA mass was remarkably smaller at one month after the administration of oral corticosteroid therapy.

showed chronic inflammatory infiltration of $\mathrm{IgG} / \mathrm{IgG} 4-$ positive lymphocytes and plasma cells and fibrotic changes, so the diagnosis of IgG4-related autoimmune pancreatitis was made pathologically. Thoracic computed tomography revealed a large mass occupying the RA (Fig. 1D). It had a round shape and a diameter of approximately $40 \mathrm{~mm}$. He had no symptoms of heart failure, and his serum brain-type natriuretic peptide (BNP) level was $44.8 \mathrm{pg} / \mathrm{mL}$. Transthoracic and transesophageal echocardiography demonstrated a huge RA mass extending from the interatrial septum (Fig. 1E and F) and no pericardial effusion. Fluorodeoxyglucose positron emission tomography showed an increased uptake in the RA mass, interventricular septum (Fig. 1B and C), pancreas, bilateral submandibular glands, thoracic wall, ascending aorta, and abdominal paraaortic lymph nodes. Coronary computed tomographic angiography revealed neither obvious stenosis nor atherosclerotic changes, although the left anterior descending coronary artery was surrounded by a tumor-like lesion with a diffuse and continuous pattern (Fig. 2A and B). We initially considered the mass to passively be a benign tumor, including cardiac myxoma, primary malignant neoplasm, or metastatic tumor. Considering the risk of pulmonary embolism, we recommended the patient undergo open chest surgery to resect the mass in RA and peri-coronary artery. However, he declined our proposal because of the risk associated with the invasiveness of the procedure. Therefore, we proposed coronary angiography and a percutaneous biopsy of the RA mass using a bioptome catheter. Coronary angiography showed no stenosis (Fig. 2C and D), but intravascular ultrasound imaging of the left anterior descending coronary artery revealed a tumor-like lesion with a low-echoic pattern extending between the media and the adventitia with a depth of 3 to $5 \mathrm{~mm}$ (Fig. 2E). We performed an intracardiac echo catheter-guided percutaneous biopsy of the RA mass using a bioptome catheter (Fig. 3A), and it showed chronic inflammatory infiltration of $\mathrm{IgG} / \mathrm{IgG} 4$-positive lymphocytes and plasma cells (Fig. 3B-D). The ratio of IgG4-positive cells/ IgG-positive cells exceeded $40 \%$, and IgG4-positive cells numbered $>10 / \mathrm{HPF}$. Therefore, we diagnosed the mass lesion as definitely IgG4-related disease. We found no evidence suggesting malignant lymphoma, such as cellular atypia or a high nuclear/cytoplasm ratio. The peri-coronary arterial mass was also considered to be associated with IgG4-related disease since some studies had reported IgG4related disease showing tumorous lesions of coronary arteries. As corticosteroid therapy was empirically effective in the treatment of IgG4-related disease, we started to treat the patient with $40 \mathrm{mg}(0.6 \mathrm{mg} / \mathrm{kg})$ of prednisolone daily. The dose of prednisolone was gradually tapered to $5 \mathrm{mg}$ daily and maintained at that dose. His serum $\mathrm{IgG}$ and $\mathrm{IgG} 4$ levels dramatically decreased over 9 weeks (IgG decreased from $2,835 \mathrm{mg} / \mathrm{dL}$ to $1,550 \mathrm{mg} / \mathrm{dL}$, IgG4 from $1,440 \mathrm{mg} / \mathrm{dL}$ to $448 \mathrm{mg} / \mathrm{dL}$ ) and the mass in the RA and peri-coronary artery had markedly shrunken within 1 month on the followup thoracic computed tomography and transthoracic echocardiography (from $40 \mathrm{~mm}$ to $25 \mathrm{~mm}$ in a diameter; Fig. 1G). No luminal narrowing of the coronary arteries was 


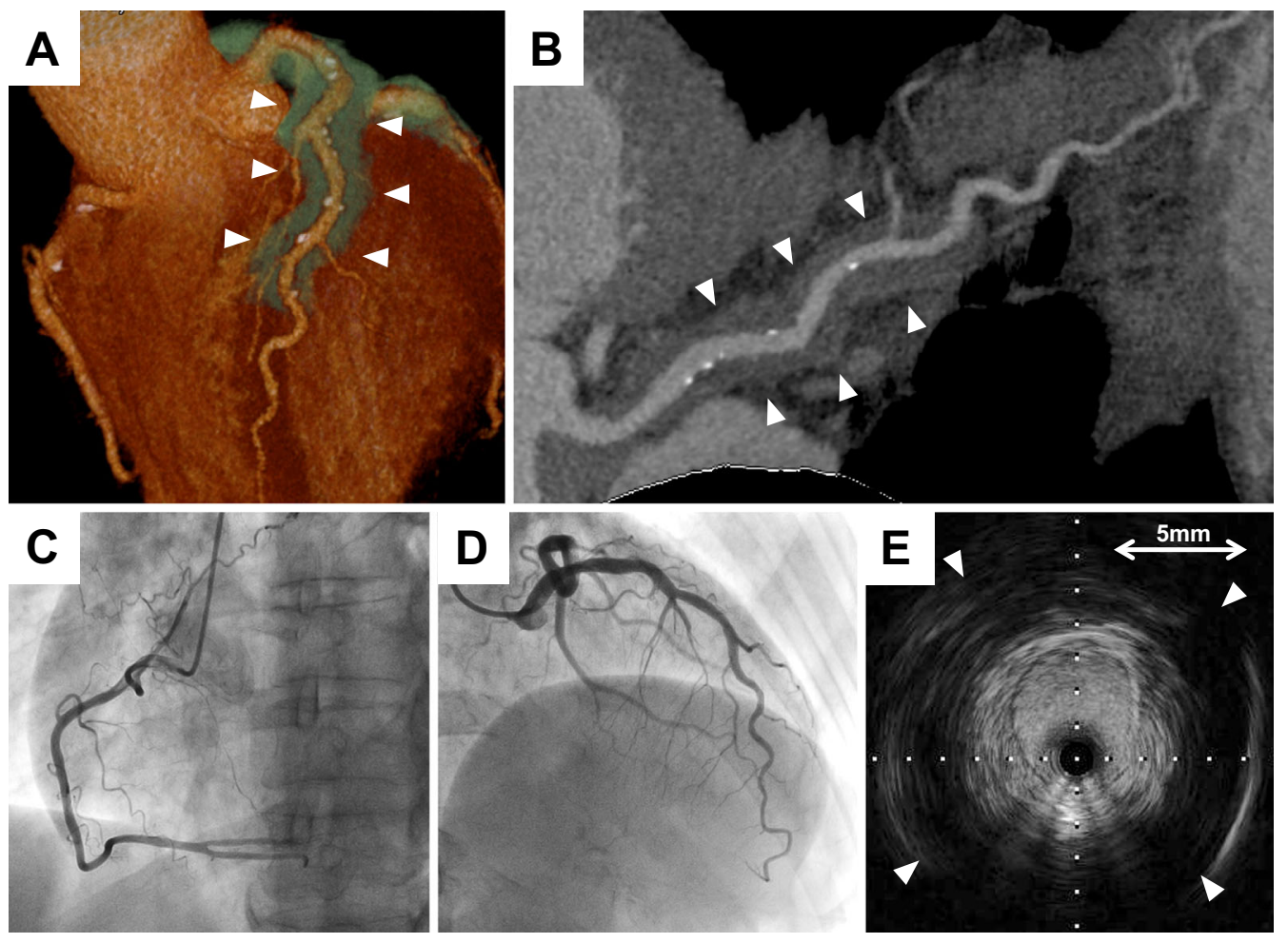

Figure 2. (A and B) Coronary computed tomographic angiography shows a tumor-like lesion surrounding the left anterior descending coronary artery. ( $C$ and $D)$ Coronary angiography shows no stenosis in the coronary arteries. (E) Intravascular ultrasound imaging of the left anterior descending coronary artery shows a large, low-echoic lesion between the media and the adventitia (arrowheads). The lesion is considered to be a tumor-like lesion on coronary computed tomographic angiography.
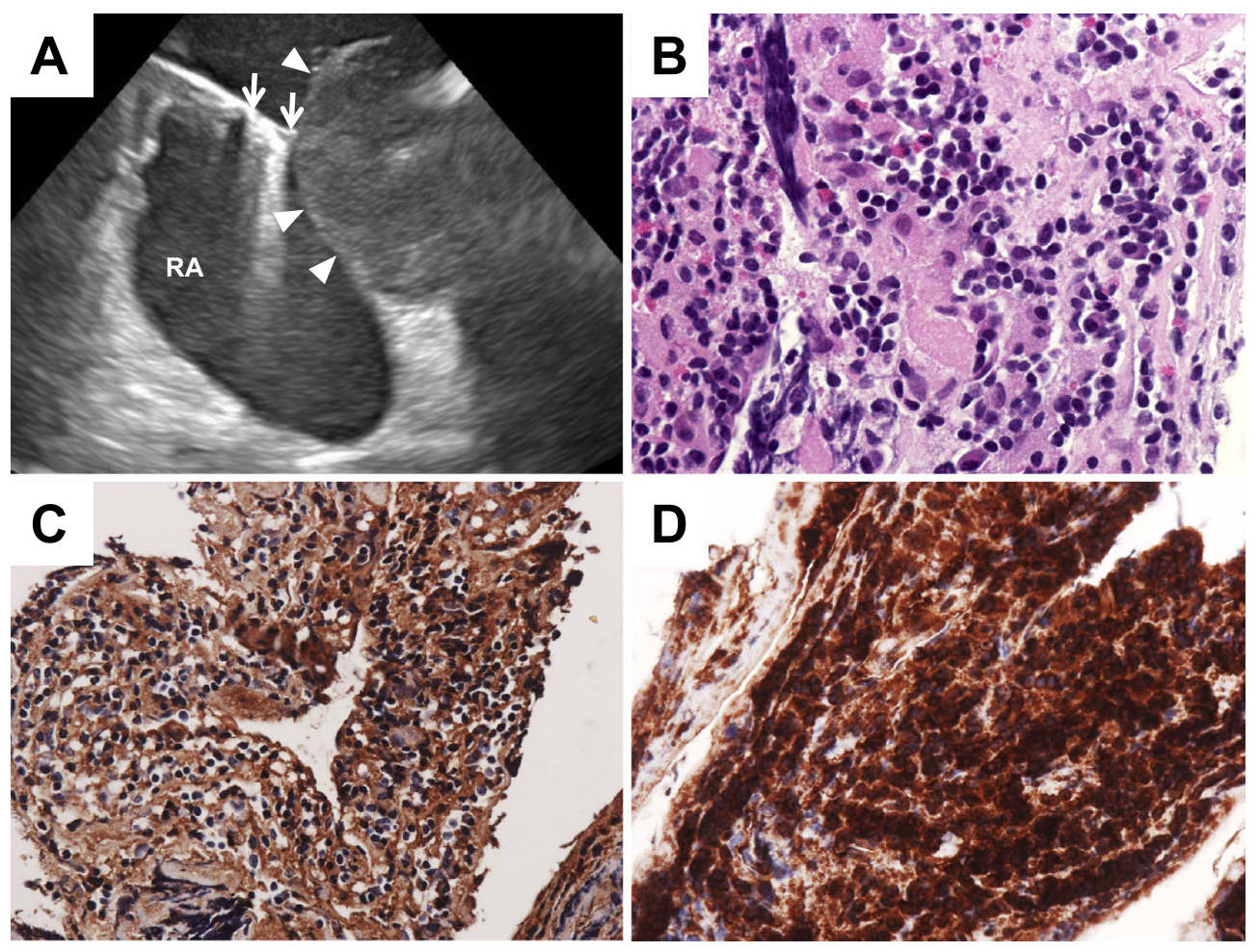

Figure 3. (A) An intracardiac echo catheter was used for the intracardiac percutaneous biopsy of the RA mass (arrowheads). (B) Many lymphocytes and plasma cells were found to have infiltrated the mass lesion (Hematoxylin and Eosin staining, original magnification $\times 60)$. (C and D) Most of the plasma cells are IgG- and IgG4-positive [immunostaining of IgG (C) and IgG4 (D), original magnification: $(C) \times 40,(D) \times 60]$. 
seen on the follow-up coronary computed tomographic angiography. He had not experienced any chest symptoms at his one-year medical follow-up examination.

\section{Discussion}

IgG4-related disease was originally discovered in patients with autoimmune pancreatitis, accompanied by elevated serum IgG4 levels $(1,2)$. Several recent studies have reported that IgG4-related disease invades every organ system, including the cardiovascular system (3), with manifestations including aortic aneurysms and lesions in peri-coronary arteries. Only case report has described an RA mass with IgG 4-related disease involving the sinus and atrioventricular node, in which the patient experienced syncope and underwent a pacemaker implantation (4). We also encountered a case with RA mass lesions that were diagnosed using an intra cardiac echo-guided biopsy. Histologically, the lesion was composed of proliferating IgG-/IgG4-positive lymphocytes and plasma cells. To our knowledge, this is the first report regarding the histological diagnosis of a cardiovascular lesion of IgG4-related disease. The mass surrounding the left anterior descending coronary artery was also considered to be associated with IgG4-related disease, since some recent studies have reported cases of IgG4-related disease showing similar tumorous lesions of the coronary arteries (5-8). There is no consensus concerning the optimal treatment of IgG4-related disease. Some cases have been treated with surgical resection of the mass or with coronary arterial bypass grafting (CABG) or percutaneous coronary intervention (PCI) when the mass surrounding the coronary artery caused luminal narrowing (5-7). We performed oral corticosteroid therapy while closely monitoring for cardiovascular events because the patient had no complaints of chest symptoms and no luminal stenosis was noted on coronary CT angiography. As a result, the therapy in the present patient markedly reduced the serum IgG4 levels to normal values and decreased the size of the mass in the RA and the mass lesions surrounding the coronary arteries. Although there have been no cases in which corticosteroid was effective for treating an intracardiac mass associated with IgG4related disease and improving the long-term prognosis, this approach might be a viable option. We believe that low-dose corticosteroid therapy should be continued, as is recommended for recurrence prevention in cases of IgG4-related autoimmune pancreatitis. In conclusion, we should be aware of the fact that $\mathrm{IgG}$-related disease can involve the cardiovascular system, which may lead to a fatal outcome because of angina pectoris, heart failure or syncope. In addition, further studies are needed to establish the optimal therapy for IgG4-related disease.

The authors state that they have no Conflict of Interest (COI).

\section{References}

1. Stone JH, Zen Y, Deshpande V. IgG4-related disease. N Engl J Med 366: 539-551, 2012.

2. Umehara H, Okazaki K, Masaki Y, et al. A novel clinical entity, IgG4-related disease (IgG4RD): general concept and details. Mod Rheumatol 22: 1-14, 2012.

3. Ishizaka N, Sakamoto A, Imai Y, Terasaki F, Nagai R. Multifocal fibrosclerosis and IgG4-related disease involving the cardiovascular system. J Cardiol 59: 132-138, 2012.

4. Song C, Koh MJ, Yoon Y, Joung B, Kim SH. IgG4-Related Sclerosing Disease Involving the Superior Vena Cava and the Atrial Septum of the Heart. Yonsei Med J 54: 1285-1288, 2013.

5. Matsumoto Y, Kasashima S, Kawashima A, et al. A case of multiple immunoglobulin G4-related periarteritis: a tumorous lesion of the coronary artery and abdominal aortic aneurysm. Hum Pathol 39: 975-980, 2008.

6. Urabe Y, Fujii T, Kurushima S, Tsujiyama S, Kihara Y. Pigs-in-ablanket coronary arteries: a case of immunoglobulin G4-related coronary periarteritis assessed by computed tomography coronary angiography, intravascular ultrasound, and positron emission tomography. Circ Cardiovasc Imaging 5: 685-687, 2012.

7. Ikutomi M, Matsumura T, Iwata $\mathrm{H}$, et al. Giant tumorous lesions (correction of legions) surrounding the right coronary artery associated with immunoglobulin-G4-related systemic disease. Cardiology 120: 22-26, 2011.

8. Kusumoto S, Kawano H, Takeno M, et al. Mass lesions surrounding coronary artery associated with immunoglobulin G4-related disease. J Cardiol Cases 5: e150-e154, 2012.

The Internal Medicine is an Open Access journal distributed under the Creative Commons Attribution-NonCommercial-NoDerivatives 4.0 International License. To view the details of this license, please visit (https://creativecommons.org/licenses/ by-nc-nd/4.0/).

(C) 2019 The Japanese Society of Internal Medicine

Intern Med 58: 2363-2366, 2019 